\title{
Mental Models, Visual Reasoning and Interaction in Information Visualization: A Top-down Perspective
}

\author{
Zhicheng Liu and John T. Stasko, Member, IEEE
}

\begin{abstract}
Although previous research has suggested that examining the interplay between internal and external representations can benefit our understanding of the role of information visualization (InfoVis) in human cognitive activities, there has been little work detailing the nature of internal representations, the relationship between internal and external representations and how interaction is related to these representations. In this paper, we identify and illustrate a specific kind of internal representation, mental models, and outline the high-level relationships between mental models and external visualizations. We present a top-down perspective of reasoning as model construction and simulation, and discuss the role of visualization in model based reasoning. From this perspective, interaction can be understood as active modeling for three primary purposes: external anchoring, information foraging, and cognitive offloading. Finally we discuss the implications of our approach for design, evaluation and theory development.
\end{abstract}

Index Terms - mental model, model-based reasoning, distributed cognition, interaction, theory, information visualization

\section{INTRODUCTION}

A deep understanding of the role of interactive visualization in human cognitive activities has been an important issue in the research of information visualization (InfoVis). Within the field of cognitive science, there has been a general movement away from focusing on internal cognitive mechanisms towards a more ecological and situated account of cognitive behavior. Influenced by this trend, researchers in HCI and InfoVis have also explored the implications of such a paradigm shift specific to visualization research. Scaife and Rogers argue that more attention should be focused on "the cognitive processing involved when interacting with graphical representations, the properties of the internal and external structures and the cognitive benefits of different graphical representations" ([48], emphasis in the original). We argued that from a distributed cognitive perspective, it is important to understand the interplay between internal and external representations and to account for the role of interaction in the emergence of cognition [30]. Despite these proposals, however, little existing work actually addresses more concrete questions such as the following:

- What is meant by the notion of "internal representation"? While external representations are intuitively understood, the term "internal representation" lacks a precise connotation. This ambiguity likely originates from cognitive science. Some cognitive scientists believe that when we perceive the world, our brains compute and transform visual sensations on the retinas into symbolic descriptions of the world [46]. Some others argue that perception should not be understood as the computation of this kind of internal representation but as the active interaction between organisms and the environment $[12,37]$. Yet some other cognitive scientists instead focus on a different genre of representations, termed schemas [34], frames [23], propositions and mental models [19]. Among these varied formulations, which are potentially useful for discussing issues in InfoVis?

- Given a clearer notion of internal representation, what are the relationships between external representations (visualizations) and internal representations? The external cognition approach

- Zhicheng Liu and John Stasko are with the School of Interactive Computing and the GVU Center at the Georgia Institute of Technology, E-mail: zliu6@gatech.edu and stasko@cc.gatech.edu.

Manuscript received 31 March 2010; accepted 1 August 2010; posted online 24 October 2010; mailed on 16 October 2010.

For information on obtaining reprints of this article, please send email to: tvcg@computer.org. suggests that "the interplay between internal and external representations in problem-solving is likely to be more complex, involving cyclical, interacting processes" [48]; such a statement is still too vague and not likely to be useful. What exactly are the complex, cyclical and interacting processes and how do we put them in concrete specifications?

- How are external visualizations, people's mental faculties and physical actions related? To our knowledge, the only theoretical framework which relates these aspects of human cognition in an integrative account is the classical perception-cognition-action model. Yet it has been acknowledged to be "less useful as a model in situations such as analytic discourse" [56] (p.51): for one, actions are not merely responses to visual stimuli [54], and cognition is more an emergent property of interaction [30]. Is it possible to propose an alternative formulation that can better inform our understandings of the relevant issues in InfoVis?

Some might dismiss the importance of these questions as they are too "cognitive" and not directly relevant to InfoVis. This way of thinking is presumably based on the assumption that a thorough understanding can be achieved by studying internal representations and external representations separately. This assumption has dominated cognitive science and still underlies a variety of fields including InfoVis. In a way, the exclusive focus on external representations in InfoVis research is an ironic parallel of the exclusive focus on internal representations in traditional cognitive science. We have argued that such an assumption should be re-examined and it is important to make cognition a research agenda for InfoVis [30]. At a higher level, answers to these questions will intrinsically contribute to a science of interaction [43] in providing an integrated view on visualization, interaction and the analytic process.

In this paper we suggest preliminary answers to the questions outlined above. To begin with, the concept of mental model discussed in the cognitive science and HCI literature seems to be relevant for describing a specific kind of internal representation in the context of InfoVis. The definition and characterization of mental model in these fields, as we argue, can be extended and synthesized to provide a useful theoretical tool for InfoVis (Section 2). With an elucidation of the mental model concept specific to InfoVis, we explore high level relationships between mental models and external visualizations (Section 3). Reasoning can be understood as constructing and simulating a mental model and we discuss how this view can shed light on the role of visualization in model based reasoning (Section 4). Interaction can then be understood from the perspective of model based reasoning, serving three primary purposes: external anchoring, information foraging and cognitive offloading (Section 5). Finally we explore the 
implications of our theories for design, evaluation and theory development.

\section{InTERnal Representations In INFOVIS: THE CONCEPT OF MENTAL MOdEL}

Although the current focus of InfoVis research is on external visualizations and interaction, it would seem implausible that no internal representations are involved in using visualizations. In particular, research in cognitive science and cognitive psychology has suggested that people have internal representations of the very visualizations they use $[15,29,57]$. It has also been argued that developing external visualizations alone cannot replace the need to understand or develop internal visualization skills, and the design of external visualizations should be informed by research on internal visualization [15]. Nevertheless, much of the related research is on internal visualizations of physical or geometric entities such as mechanical systems or geometric shapes, and has not touched a core aspect of InfoVis - the representation of abstract information. Furthermore, the term "internal visualization" does not seem to be sufficient for InfoVis considering the complexity of the issues involved including interaction, data-visualization mapping and information about data conveyed through visual metaphors. As a first step to investigate internal representations in InfoVis, we argue that the mental model framework in cognitive science and $\mathrm{HCI}$ may provide a useful theoretical concept.

\subsection{Mental Models in Cognitive Science and $\mathrm{HCl}$}

The term "mental model" is likely familiar to most of us, and it has been used as a common term in everyday communication. In academic contexts, the term was first proposed by Craik [7], whose work is now considered as a cornerstone for studies on human cognition. Research work on mental models is extensive and spans across disciplines and domains. Although it is generally agreed that a mental model is a "small scale" internal representation of a real-world phenomenon [7], the usage of the term across the related disciplines carries different emphases and meanings, which are not always directly transferable to InfoVis. Payne argues that the term is used in so many different ways that the idea of a mental model may have contributed to the decline in interest in theoretical approaches to HCI [42]. Rouse and Morris also observe that despite the ubiquitous use of the term, few explicit definitions are formulated. They argue that a pragmatic investigation on mental models in application domains is necessary [47].

Our first goal in this paper is to devise a reasonable and usable definition of mental model specific to InfoVis research. Reviewing existing literature, two influential and potentially relevant strands of research stand out: in cognitive science, the theory of mental model was expounded by Johnson-Laird [19] and has been systematically used in investigating the role of mental models in logical reasoning and language comprehension; in the field of HCI, among other pioneering researchers, Norman worked on extending the concept of mental model to investigate users' conceptions of how a system works [38].

Norman argues that a fundamental characteristic of mental models is that they must be functional [38]. Mental models do not have to be and usually are not accurate, but they must be "runnable" to serve certain purposes. Norman's discussion, based on observed calculator usage, mainly focuses on users' beliefs about the behavioral aspect of a system, e.g. what happens when the CLEAR button on a calculator is pressed. Having a mental model of the system implies that users have conceptions about the system's inner working. Along this line of thought, Kieras and Bovair define a mental model of a device as "how a device works in terms of its internal structures and processes" [21]. This strand of research in general does not explore in depth issues such as how a mental model is represented or the nature of its processing and format.

Johnson-Laird's theory of mental models is more elaborate [19]. He agrees with the functional characterization of mental models, but puts a stronger emphasis on the structural aspect. His theory was a critique of the view that thinking and reasoning are inherently governed by and based on formal logic. To him, mental models are analogues of what they represent, preserving relations between entities. People construct and manipulate mental models in reasoning so that the conclusion can be directly read off without presupposing mental logics and formal rules. Mental models are created in working memory, usually in the visuo-spatial sketchpad [32] with a limited capacity.

Due to the nature of problems being investigated and the difference in emphasis, Johnson-Laird's notion of mental model seems quite different from Norman's. For example, in a simple reasoning task with premises Tom is older than Kate and Tom is older than Bob, reasoners can construct two different models in working memory where the relations between entities are represented as spatial relations:

$\begin{array}{lllr}\text { model } 1 & \text { Tom } & \text { Kate } & \text { Bob } \\ \text { model } 2 & \text { Tom } & \text { Bob } & \text { Kate }\end{array}$

Both models are consistent with the premises and reasoners know that the genealogical order between Kate and Bob cannot be determined by simply examining their mental models. People do not actually represent the premises in terms of logical predicates such as older (Tom, Bob) and apply logic rules to arrive at a conclusion.

\subsection{Relevance and Applicability for InfoVis}

These formulations of mental models can serve as important groundworks for thinking about internal representations specific to InfoVis. First of all, the notion that mental models must be functional is appropriate as visualizations, whether internal or external, are tools for reasoning. It is generally agreed that different visual representations have different affordances for certain data types and tasks. Norman's focus on behavioral aspects of mental models can be easily related to how an InfoVis system works, especially via the built-in interaction techniques. Johnson-Laird's emphasis on the structural analogy is important too as an essential function of InfoVis is to reveal spatial, temporal or distributional relations among data items. There are, however, several issues that must be resolved before mental model can become a truly useful concept for InfoVis, which we address below.

\subsubsection{Model or Image}

Applying Johnson-Laird's theory to InfoVis touches on a thorny issue regarding the format of mental models. Johnson-Laird argues that mental models are more abstract and are basically spatial representations. This observation is made in contrast with mental imageries [26], which are believed to be richer in color, texture and form. Furthermore, mental models can contain text symbols and mental imageries do not [20, 24]. Johnson-Laird does acknowledge though that both mental models and imageries can be used in reasoning.

Such characterization makes it difficult to apply the concept of mental models to InfoVis, although Johnson-Laird himself is not totally consistent on this issue. In InfoVis, spatial layout is only one visual cue and is often used in combination with other cues such as hue and size. It seems unreasonable to draw such a clear distinction. Our own experience indicates that the mental model of an InfoVis system is likely to be in the format of a "cognitive collage" [59], where text, images and coarse spatial relations are overlaid. In the process of designing SellTrend [31], at one point we faced the problem of determining whether we should keep the coloring of an attribute value constant throughout user exploration or we should update it every time users apply or remove a filter. Since making the decision involved potential features that had not been implemented, one of the authors (A1) constructed a mental model of the ongoing SellTrend system and used it in his simulative reasoning while thinking aloud:

A1 considered the case of keeping the color unchanged first: "I remember there is an error code $* * *$, it usually has the highest percentage, now if I move it to the exclusion filter", [moving his fingers from right to left in the air, corresponding to the filtering action to be performed on the actual interface], "the next one (error code) got blown up, and it's almost if we want it to stay light red, instead of 
dark red; now it would be weird 'cause somehow we want to change the denominator too (Equation 1, [31])."

Then he considered the case of updating the color: "in another case, let's say an airline is closely associated with a SID (agent), in the initial world state the SID is colored in light red, now I drag the airline into the filter, I will want to see dark red instead of light red."

Finally he commented that it would be better to implement these two alternatives, see them in action and talk to the company analyst about it.

It can be argued that in the mental models constructed, the spatial, chromatic and textual representation of data attributes are all preserved and used in the thinking process. To be able to talk about issues in InfoVis, we contend that the scope of mental models should be extended to include other visual properties. After all, as some argue, the distinction between mental models and visual imageries may be attributed to an overly restricted use of the term mental model [13] and the distinction between models and imageries is far from a settled issue.

\subsubsection{Mental Simulation}

Since theories on mental models in HCI do not elaborate on the format issue, little is explicated about the working mechanisms of mental models. Work in cognitive science tends to pay more attention on this issue. Nevertheless, some argue that due to Johnson-Laird and colleagues' focus on logical reasoning tasks and the distinction between mental models and imageries, the concept of mental simulation as a central working mechanism of mental models in reasoning is underplayed [36].

To understand what it means to mentally simulate, subjective introspection can provide first-hand experience; alternatively, ethnographic accounts are often used as the basis for third-person interpretation. As discussed earlier, existing work on mental models, as epitomized by Johnson-Laird and colleagues' research on model-based logical reasoning, focuses primarily on spatial manipulations as simulations. Simulation however can be much more than changing spatial configurations, as demonstrated by evidence from domains such as engineering [11] and research on discourse models and mental animation [36].

Since we extend the concept of mental model to include other visual properties and the behavioral aspects of visualizations, simulation on mental models of InfoVis can go beyond spatial reconfiguration too. Referring to our experience described in Section 2.2.1, A1 constructed two mental models of the SellTrend system and performed simulation on each model. In this case, his tacit and explicit knowledge of the problem domain (airline reservation and purchase transactions), of the underlying data (temporal categorical event sequences) and of the system logic (parameter computation and visual mapping) enables him to perform manipulations on his mental models and create new model states. Simulation here involves not only re-arrangement of tokens spatially, but also changing the underlying system logic and behavior. This way of understanding visualization-enhanced reasoning is an exemplar of what can be termed "model-based reasoning" [33, 36], where the entire reasoning process can be explained just in terms of the construction and simulation of mental models.

\subsubsection{Tool vs. Data}

The structural, behavioral or functional characterizations of mental models seem to be tightly related to features of an InfoVis system as a tool. With this connotation, what is represented in a mental model may include the mapping schemes from data cases to visual items, the interaction techniques built into the visualization and how one might use and interact with the visualization. These are higher level "meta" features of visualizations. One question remains whether the underlying data is part of a mental model. In particular, Purchase et al. identified "mental maps" [45] as information-bearing internal representations. When a user observes and understands the layout of a graph, she creates an internal representation of the information about the data as conveyed in visual forms. It is argued that important information about the data is preserved in mental maps and dynamic graph layout algorithms should try to keep the external layout consistent with users' mental maps.

Is a mental map the same as a mental model? One way to think about this question is to ask whether information about the data is preserved in these two kinds of internal representations. Since visualizations are not really meaningful without any reference to the underlying data, it seems reasonable that mental models do preserve information about the data. What is meant by "information" here, however, deserves more careful analysis. Given a data set, multiple levels of description can be formulated regarding the information contained in the data. First, there is the schematic level information. The data set can be relational, hierarchical or more heterogeneous. Secondly, there is the semantic level information. The data underlying SellTrend [31] and LifeLines 2 [63] can both be considered temporal categorical. Due to the domain specific semantics, the data analyses and the relevant tasks to be supported differ significantly and hence require different visual representations and interaction techniques. Lastly there is the item level information. This level concerns the individual and aggregated information about specific data items. Examples include the frequency of a particular error code as shown in the scenario earlier, or the specific layout of a graph.

The term "mental maps" as used in the existing literature seems to be primarily related to item level information. A mental model may preserve item level information but does not have to. Given a data set with schematic and semantic level descriptions, a proficient user can construct and simulate mental models of different visualizations to get a feel of which visual representation may be suitable. No item-specific information has to be involved here. We thus believe that what distinguishes mental models from mental maps is that 1) mental models can be more abstract than mental maps in that they preserve high level information about a data set, and 2) mental models are more internal representations of visualizations as tools and must be functional.

\subsection{Mental Model: A Definition for InfoVis}

We thus reach the following definition of mental model in the context of InfoVis:

A mental model is a functional analogue representation to an external interactive visualization system with the following characteristics:

- The structural and behavioral properties of external systems are preserved in mental models.

- A mental model can preserve schematic, semantic or itemspecific information about the underlying data.

- Given a problem, a mental model of an interactive visualization can be constructed and simulated in working memory for reasoning.

\section{The Dynamics between Mental Models and Exter- NAL VISUALIZATIONS}

Existing work on mental models often focus on models as held in working memory, although they can be certainly externalized into fully developed models. The example of a mental model described in Section 2.1 can be seen as a rudimentary form of information visualization. Not much attention is on how mental modeling engages external resources. In the real world however, external representations are constantly involved in reasoning processes.

On the other hand, it has been argued that external visual formalisms, not mental models or metaphors should serve as a basis for interface design [35]. The external cognition approach [5] represents such effort to design and develop elaborate visualizations. An exclusive focus on external cognition however runs the risk of hinting that external visualizations can replace or substitute mental models, a view which is not necessarily true [15].

A more ecological perspective that examines the interplay between mental models and external visualizations has been missing. Several 
issues are at stake here. First, unlike simple spatial configurations described in Section 2.1, a mental model of visualization is complex enough to demand an explanation of how it came into being. Secondly, the externalization processes are not usually performed by the reasoners themselves. Hence the relationships between mental models and external visualizations are not just a simple inside-out or outsidein process, but more confounded. Ultimately this raises the question of how mental models and external visualizations are related. In addition, a focus on either mental models or external visualizations tends to marginalize interaction, a core phenomenon yet to be understood [66]. This ties back to the third question we have raised in the introduction. An integrated account of mental models, external visualizations and interaction in the reasoning process is needed.

In this section we consider four possible high-level dynamics between mental models and external visualizations: internalization, processing, augmentation and creation (Figure 1). We briefly summarize each of these relationships, and will elaborate on augmentation and discuss how the concept of mental model can lead to new ways of understanding interaction in visual reasoning in the following sections.

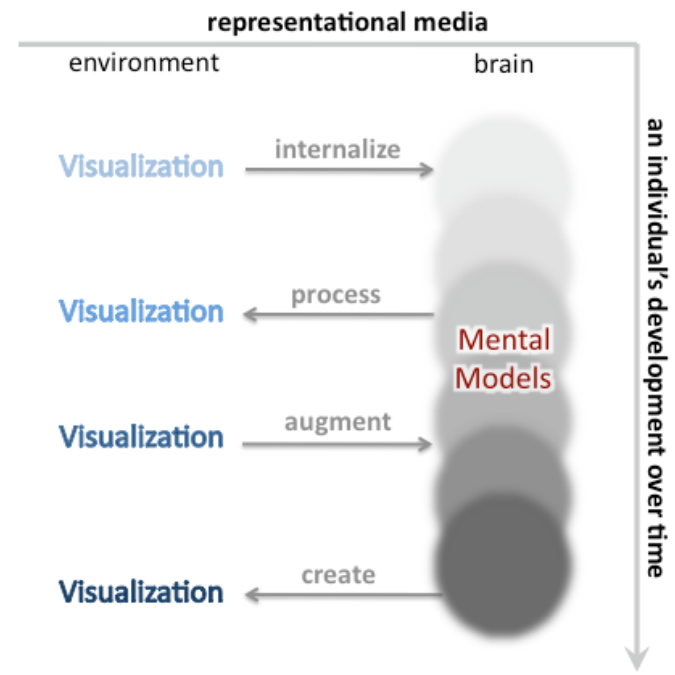

Fig. 1. Visualization as an interactive process across representationa media: a developmental perspective

\subsection{Internalization: Mental Models as Abstractions of Vi- sualizations}

Following the previous discussion, an obvious kind of relationship between mental models and external representations is internalization. The formation of a mental model happens ontogenetically after the appearance of the original external phenomenon. This insight has been captured and formulated by Vygotsky, who argues that in each individual's development, every higher order cognitive function appears twice: first between people, as an inter-psychological process, then inside an individual, as an intra-psychological process [62, 64]. In InfoVis, it makes sense too to understand the role of visualization in human cognitive activities from a developmental perspective.

Using cognitive tools such as visualization is not an innate ability. For anyone who has never seen an InfoVis system before, interpreting and using the visualizations is difficult. The first stage of development hence is learning, which involves the interaction between potentially the designer and the user through the visualization on a social plane. There are significant differences in the learning processes whether the user has had experience with other InfoVis tools or she is a complete novice. In this inter-psychological interaction involving InfoVis, not only the property of InfoVis matters, the prior experience of the user, the mediating signs used such as language and gesture, and even the environmental settings all play a role in influencing the outcome of the learning process.
Learning, if effective, results in the mastery of the InfoVis system. From a cognitive point of view, this means the successful internalization of the system as a mental model. We assume that internalization involves the encoding of information abstracted from perception in long-term memory. Since it often does not make sense to assume independent existence of visualizations and their underlying data, information about the data, especially at schematic and semantic levels, may be preserved at the same time. The mental models need not be veridical or isomorphic to the corresponding external visualizations [15], but they must be functional.

\subsection{Processing: Mental Models as Processors of New Vi- sualizations}

Mental models internalized can in turn process and make sense of new external phenomena including visualizations. Ziemkiewicz and Kosara [67] investigated how the compatibility (or lack thereof) between users' mental models and hierarchical visualizations can influence performances in using visualizations. Hierarchical data can be described in different external representations either as levels or containment, using words or visualizations. These different ways of representation imply different structural properties, which are preserved in the internalized mental models per our definition. Through priming, a mental model may be constructed in working memory that is not in accordance with the external representation structurally, interference can happen and affect performance.

There has been virtually no study on how users understand new external visualizations. Potentially related research examines how people comprehend stories by constructing mental models of the situations depicted by the narratives [19].

\subsection{Augmentation: Mental Models as the Basis for Rea- soning using Visualizations}

Mental models of internalized visualization can be instantiated and simulated in reasoning and sensemaking. In most cases, however, pure mental modeling is not sufficient; the availability of external visualizations can augment internal visual models so that external representations are integral to a distributed and coupled cognitive system. Since most of the InfoVis research is concerned with using visualization in supporting sensemaking and reasoning, we will focus on this particular relationship and elaborate further in the rest of this paper.

\subsection{Creation: Mental Models as the Basis for Novel Visual Design}

Finally, mental models can serve as the cognitive basis of creativity and innovation. The construction and simulation of mental models can give rise to new concepts and designs including novel visual representations. Some visual representations such as the scatter plot and line graph have existed for centuries. Being internalized by visualization designers, they likely serve as the foundation for creating novel visual designs such as parallel coordinates and theme rivers. Although there has been few studies focusing on the role of mental models in the creation of new visualization designs, studies in domains other than InfoVis reveal interesting insights on how new innovations and discoveries are often not made as a result of logical inference or deduction, but via mechanisms such as analogy and simulative modeling $[11,18,36]$.

It should be noted that interaction plays a key role in all these cases, although we will only discuss interaction in model based reasoning in this paper. In addition, the formulation of these different kinds of relationships does not imply they are independent; instead, these processes often take place together and are interweaved. For example, in processing and interpreting new visualizations, the new visualizations are likely being internalized at the same time.

\section{Model based Reasoning}

\subsection{Modeling in a Distributed System}

As discussed in Section 2.2.2, a primary function of mental models is that given a problem, they can be constructed and simulated in hu- 
man working memory for reasoning, anticipation and planning. The scenario presented in Section 2.2.1 is of course not a representative use case of visualization. Visualization is primarily designed to make sense of data and attain insights. In addition, there is a difference in terms of where the visualization resides: in the scenario the visualization is internal, while visualization is usually external in data analytics. Despite these differences, however, we hypothesize that the roles of InfoVis and the working mechanisms of mental models in purely mental simulative thinking and in the process of making sense of data are similar. In both cases, visualization is not just an input or scaffold for human reasoning; people construct and manipulate models of visualization so that they are something to think with. Similar hypotheses have been put forward in the cognitive science literature too. For example, in their seminal article, Larkin and Simon claimed "mental imagery - the uses of diagrams and other pictorial representations that are not stored on paper but are held in human memory ... play a role in problem solving quite analogous to the role played by external diagrams" [29] (p.97) .

From this point of view, the boundary of the cognitive system that implements the visualization is very flexible. In A1's case, the model is in the head; its construction and manipulation is achieved primarily by mental simulation, although parts of human body are involved too (e.g. A1's gesture to enact a filtering action). In the case of making sense of the data, the model expands the boundary of the cognitive system from the brain to include external visualization. Users thus do not have to hold the entire model in their heads: the world serves as "its own best model" [4]. The model can be implemented in a distributed system comprising of internal or external media and thus has a fluid boundary - a perspective we have advocated [30].

In a way, expanding the boundary of the cognitive system is indispensable for data analytics. First, human working memory is known to be limited. A complex model of the InfoVis system and relevant knowledge cannot be fully constructed in detail in working memory. Secondly, what makes an InfoVis system work is the physical precision of visual cues computed algorithmically, no mental modeling can achieve a comparable precision and thus must be carried out with an InfoVis system at hand. To be able to make sense of data however, mental simulation alone is even more insufficient because per our definition, mental models do not have to contain item specific information about the data; external data provides essential information source to enable sensemaking.

\subsection{Two Perspectives}

The formulation of model based reasoning hence is radically different from the traditional view in cognitive science. Such differences have implications on what questions should be asked and what constitutes an understanding of the role of visualization in reasoning. In the traditional cognitive science view, visualization is an input to the central cognitive processor, equated with the brain, and physical actions carry out outputs from this processor. Understanding the role of visualization in reasoning means uncovering the working mechanisms of the brain, which are often assumed to take the form of algorithmic computations.

Model based reasoning turns this view inside out. Visualization might be considered as an input in that it is internalized as a mental model. The working mechanisms of a mental model however are likely not digitally orchestrated, but may be in the "organic" form of analog simulation. Theoretically, mental model based reasoning can take place without any external representation. In actual data analysis, however, external visualization is needed to augment internal visualization models [15], and the boundary of the cognitive system expands accordingly so that external visualization is integral to the system. Interaction with external visualization is analogous to mental simulation confined in one's head, both involve the construction and manipulation of visualization for simulative reasoning, the difference is that the former involves not only mental simulation but also the active participation of the human body. Interaction is not merely a delegate or executor of the cognitive processes in the head, it is a central part of the cognitive process.
Understanding the role of visualization in reasoning hence places the focus not on the brain, but on interaction. We thus need to understand what is the difference in terms of model construction and manipulation between pure mental simulation and modeling actions in a distributed system. In specific, what exactly are the roles of different parts of the human body in interacting with visualizations in the analytic process, and how can we relate observable behavior such as eye movements and mouse clicks to conceptual characterizations of model construction and manipulation? Now that we have set up the framework of model based reasoning, we can examine these questions in depth.

\section{Interaction in Visual Model based Reasoning}

\subsection{Beyond Interaction Techniques}

Most of existing work on interactive aspects of InfoVis focuses on interaction techniques, which are both actions initiated by humans and features built into the visualization (e.g. $[8,28,60,65,66])$. Generally speaking, interaction techniques can be characterized as 1) involving physical motor actions, and 2) resulting in change in the state of visualization. This approach, however, fails to capture the diversity of human actions in interacting with visualizations. Tufte's formulation demonstrates such diversity:

\begin{abstract}
"We thrive in information-thick worlds because of our marvelous and everyday capacity to select, edit, single out, structure, highlight, group, pair, merge, harmonize, synthesize, focus, organize, condense, reduce, boil down, choose, categorize, catalog, classify, list, abstract, scan, look into, idealize, isolate, discriminate, distinguish, screen, pigeonhole, pick over, sort, integrate, blend, inspect, filter, lump, skip, smooth, chunk, average, approximate, cluster, aggregate, outline, summarize, itemize, review, dip into, flip through, browse, glance into, leaf through, skim, refine, enumerate, glean, synopsize, winnow the wheat from the chaff and separate the sheep from the goats. " [58] (p.50)
\end{abstract}

Many of these actions have no counterparts as features of visualization, and they have been largely ignored in InfoVis research. One way to address this concern is to group these actions and differentiate them from interaction techniques. Spence, for example, defines "passive interaction" as interaction not involving physical motor actions, where prototypical exemplars are eye movements [52]. Categorizations such as this are often based on modality (which part of human body is involved) or on characteristics of visualization (static or interactive).

From a human-centered perspective, however, these bases of distinction do not seem to be robust. Consider an example where a user wants to explore neighboring visual items of the currently selected item. On a small screen, she will have to pan and zoom; on a large screen, however, she only has to turn her head slightly and move her eyes across the screen. In the former case, interaction is clearly in the form of interaction techniques; in the latter there is only "passive interaction". In both cases, however, these seemingly distinct behaviors can be understood by the same underlying intent [66]. It seems unjustified to treat actions such as eye and head movements as qualitatively different from interaction techniques simply because of the difference in modality. Despite the efforts to recognize those often ignored human actions, we are left with a disjointed picture of human performance.

\subsection{A Holistic Approach to Interaction}

The process of model based reasoning consists of a variety of tasks. Depending on what environmental resources are available, there can be many different ways of accomplishing a task. For example, the task of schematizing may be accomplished only in the head of the analyst, using pen and paper, or through a more elaborate external representation supported by the computer interface [44]. As discussed in Section 4.1, from a cognitive point of view, this implies a dynamic change of the boundary of the cognitive system in which a mental model is 
constructed and manipulated. Apparently the affordances and constraints of the tool come into play and shape the course of action, and the distribution of representation across internal and external medium dynamically changes.

The expansion of the boundary of the cognitive system does not imply that all the mental activities are completely transferred to the external plane, rendering the internal plane irrelevant. Even with a manipulable external visualization, expert users may still rely extensively on internal visual models. For example, Trafton et al. [57] report that scientists perform many mental operations including spatial transformations on their own mental models as well as external scientific visualizations. In addition, as we have discussed earlier, mental modeling not only is based on internalized visualizations but also involves activated past experience and knowledge that are relevant in the current context. This past experience or knowledge is still represented internally and arguably in the form of frames, schemata or propositions.

As a result, when internal visualizations are augmented by external ones, additional problems are created: when we are simulating the model purely in the head, there is nothing inherent that stands in the way of integrating the model with the thinking process. The blatant separation of the internal and external realms implies efforts are needed to bring them together to form a coupled system. Ideally, such a distributed yet coupled system should enable seamless information flow between the human and visualizations while minimizing the $\operatorname{cog}$ nitive work that has to be performed internally or interactively.

With this observation in mind, and considering the two major inadequacies of pure mental modeling we have discussed earlier (1. lack of precise information in mental models, and 2. limitations of human working memory), we propose the primary functionalities of interaction are threefold : to enable external anchoring, information foraging and cognitive offloading. We elaborate this view in this section by categorizing and interpreting canonical examples of human actions performed on visualizations.

In a similar vein to previous work on interaction $[14,66]$, we emphasize human intentionality as a basis for understanding interaction in InfoVis. Our approach differs from existing work in that we do not limit ourselves to consider only interactive features of InfoVis systems; instead we take a human-centered perspective to look at a broader range of intent-based human actions. We elaborate the functions of these actions in the analytic process. These conceptualized actions are often carried out by low-level habitual operations that are often performed without conscious deliberation. Examples of operations include mouse clicks, eye movements and typing on keyboards. We discuss how the same action can be accomplished by different combinations of lower level operations depending on the environmental affordances and constraints. It should be noted that we do not intend to provide an exhaustive taxonomy of actions, as we are limited by the paper length as well as the diversity of actions.

\subsubsection{External Anchoring}

Project: Hutchins remarked that reasoning processes require stable representations. One pervasive strategy used by people to achieve such a stability is through the blending of conceptual structures with material anchors [16]. Similarly, Kirsh observed that people could see something extra by augmenting and projecting onto the thing present [22]. In InfoVis, projection can be considered as a way giving meaning to an external representation. Projecting is an essential kind of action in InfoVis, and it is done by mental simulation accompanied with eye fixation and movements. Colorful shapes and their arrangements on the screen are meaningless to someone who does not know what they represent. That is, the user must be able to project mental structures onto the visual forms in constructing a coupled model. In InfoVis, it is common to use additional material anchors, such as axis ticks and text labels, to facilitate projection.

External anchors of projection do not have to be totally persistent and stable. In the case of GapMinder [1] where a highly spontaneous animation takes place, if we want to keep an eye on both China and India, we would create two internal structures (visual indices) representing these two countries and project them onto the biggest two circles. Here knowing the visual encoding rule (size of the circle represents population size) is prerequisite to proper projection.

Locate: When a person uses a pencil to draw diagrams in solving a geometric problem, the external representation is created by herself and the act of externalization is tightly coupled with the action of projection. In InfoVis, however, external representations are usually generated algorithmically; hence it often takes extra effort for users to locate the appropriate representational anchors for internal-external coupling. The action of locating is initiated if the user generates a conceptual structure and needs an external representation to anchor it. Locating can be carried out by eye scanning across the screen if the target visual item is labeled, a coordination of eye scanning and mouse-over to see the tooltips of visual items, or typing a search query and a button click.

\subsubsection{Information Foraging}

Restructure: Fortuitous interaction can sometimes result in unexpected discovery because physical action is often cheaper and more precise than mental modeling. In piecing together a jigsaw puzzle, a commonly used and effective strategy is to rearrange the pieces semi-randomly. In InfoVis, interesting patterns or relationships inside the data are often revealed when a new sorting order, a new color mapping or a new visual representation is applied to the same data. Restructuring sometimes is done purely randomly; in other cases, it may be preceded by a mental simulation [57]. Restructuring includes the actions under the categories of reconfigure and encode in Yi et al.'s work [66]. Although reconfigure and encode result in considerably different ways of change in the visualization, from a human-centered perspective, they are both for the purpose of revealing information about the data that is only explicit with a different way of presenting the data.

Explore: In the course of sensemaking, sometimes the available information at hand is insufficient to make a hypothesis; at the same time, there may not be evident environmental cues that guide information foraging. In this case, users may adopt a semi-random behavior by exploring the environment to find potentially useful information. The primary difference between the action of exploring and locating is the latter has a more or less defined target and the former does not. Exploring can be accomplished by eye and head movements, or a coordination of eye movements and zooming/panning.

\subsubsection{Cognitive Offloading}

Create: During the process of visual data analysis, interesting entities, discoveries and insights can be given stable external forms in marshaling environments such as shoeboxes. The action of creating is different from projecting in that the material anchor is generated and specified by the user. In general, creating stable representations of internal structures help to reduce the amount of information kept in working memory. As Ballard et al. point out, the external world is analogous to computer memory [3]. In computer memory, dynamic pointers are generated to refer to symbolic representations containing rich information at a specific location. The execution of a program often employs the strategy of creating and destroying pointers to the symbolic representations on the fly. Such a strategy is efficient because the symbolic representations do not have to be copied or duplicated in their entirety. Similarly, by creating material anchors, we can dynamically generate deictic or pointing structures in our heads, hence reducing the load on working memory.

Save/Load: Internal pointing structures can refer to units of information larger than entities, hypotheses and evidence. The state of an entire visualization and even system can be referenced by pointers too. Depending on whether and how the system provides such functionalities, users can explicitly save or bookmark a state of a visualization or system for later retrieval; alternatively the system can automatically 
log the state every time a physical action is performed, and users can revisit these saved states using undo and redo features.

\subsubsection{The Path of Actions}

The kinds of actions described above form the building blocks of continuous interaction with visualizations. Kirsh speaks of a projectcreate-project cycle as the interactive process in reasoning and sensemaking [22], where "project" corresponds to the external anchoring actions and "create" corresponds to cognitive offloading actions. People constantly use external representations to facilitate projection and mental simulation. Information generated is in turn created or externalized to free up cognitive resources. The process of creation at the same time changes the external representations to make it easier to project further. In InfoVis, however, information is not only generated in the head as insights but also extracted from data sources. This project-create-project cycle hence needs to accommodate information foraging actions. We thus extend Kirsh's formulation as depicted in Figure 2.

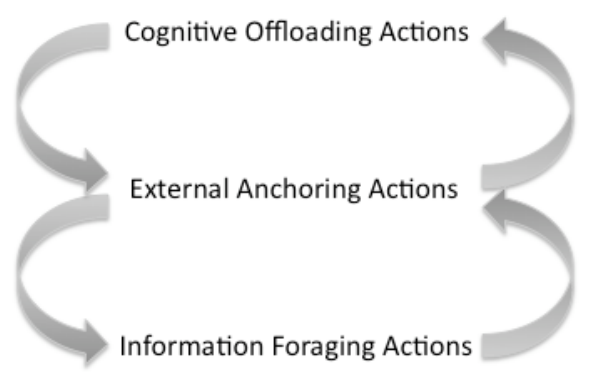

Fig. 2. Cycles of human action in using visualizations for model-based reasoning

For example, in a situation where the screen is visually crowded or cluttered, locating can be difficult. Even with successful locating, a shift of attention or a change in the visualization state can result in difficulty in projecting. Users thus often take advantage of the select or mouse-over function usually built into visualizations, and highlight the visual item(s) of current interest. This cognitive offloading action of highlight saves users time and effort by turning locating into an action of looking. The visual representation is simultaneously changed for easy locating and projecting.

Simiarly, automatic layouts sometimes do not result in a welldesigned graph visualization judged from Gestalt principles of "closure" and "proximity", making it difficult to project conceptual structures such as cluster or group. End users can spontaneously arrange the nodes to generate layouts that they feel best capture the relationships in the data $[9,61]$. The cognitive offloading action of arrange in this case gives a stable form of the relationship between items, which facilitates projecting and mental simulation later.

When this cycle comes to a halt as users get stuck, or require additional information, information foraging actions are performed to uncover potentially new information from visualizations.

\section{Implications For DEsign \\ 6.1 Designing as Model Enculturating}

Designing for InfoVis is a process of creating visualization by giving form to one's mental simulations. Although we have not substantially addressed the process of creation in InfoVis in this paper, a first look hints that it may bear significant similarities to model based reasoning. When we are designing for a specific user group with the pertinent problems and tasks identified, we likely engage in substantial mental modeling by constructing and simulating visual representations. Again, integrating external structures is crucial: sketching on paper and boards, drawing using computer software and building prototypes on sample data are usually indispensable methods in the design process. These material structures as externalized models can be in turn internalized by users. The entire process of design hence can be considered as the propagation of visual models across individuals in a larger social system.

The success of a design can be measured in many ways. In user centered design, users' final adoption is strong evidence of the design's success. Communication between the designers and the users is the key factor to help users build up a mental model that is consistent with designer's representational encoding. In a user-centered design process, designers try to attune to the users' mental models by understanding their practice and culture; the users in turn become accustomed to the InfoVis design and provide feedback that leads to refinement of the tool. In the long run, the mental models of both parties converge.

If a design is intended for a larger user population, its success will be measured by the extent to which it is adopted. In such a case it is not practical to promote a close and long term interaction between the designers and the users. Asking what makes a design successful is then inherently asking what makes easy propagation of visual models so that they are not just material anchors for one individual, but shared culturally. Sperber hence proposed an "epidemiology of representations" [53], which focuses on explaining why some representations are more "epidemic" than others.

One possible explanation provided by research on mental models is the use of cultural models in these designs. As Shore argues, mental models are layered and best thought of as having polyphonic structures [51]. Although mental models can be highly idiosyncratic, there are conventional aspects that can be shared by groups of individuals as cultural models. In particular, there is a kind of cultural model called "foundational schemas" that serve as abstract cognitive models that form the basis of our understanding of the world. Lakoff and Johnson [27] identified some examples of such schemas generated through metaphorical extensions from bodily experiences. The up-down schema (e.g. as manifested in linguistic expressions such as I'm feeling up vs. I'm depressed), for example directly derives from the relationship between our bodies and the environment. They in turn structure and organize our thoughts in a culturally shared way.

These foundational schemas might help us understand issues in InfoVis. Shneiderman's mantra "overview first, zoom and filter, then details-on-demand" [49] is a useful design principle embraced by many designers. Its reliance on orientational metaphors is quite evident. These spatial orientations arise from the fact that we have bodies that are uniquely human and function as they do in our physical environment. By elevating and lowering our bodies, our field of vision changes. The particular way human visual system works and how our body is structured result in a more complete view of the physical environment but a loss of visual details. The mantra can be understood as rooted in this kind of foundational schemas that are derived from bodily experiences, which are intuitive to understand. Designing for the masses likely will need to discover and take advantage of these cultural models.

\subsection{Physical Constraints}

The benefits that external visual representations provide are essential: as stable physical structures, they afford external anchoring, cognitive offloading and information foraging. The constraints of such a physical environment, often constructed by designers, should not be overlooked. If users' actions are indeed analogous to mental modeling through simulation as we hypothesized, they are inherently active, intentional rather than reactive and passive. More importantly, like mental simulation, they can involve wanting to manipulate visualizations in ways that are not supported due to the constraints built into the system.

The concept of "semantic distance" [17] hence is important in the design of InfoVis systems. Semantic distance concerns the relation of the meaning of an expression in the interface language to what the user wants to say. Two important questions about semantic distance in visual based reasoning are 1) Is it possible to ask questions as intended by the analysts using the interface? That is, does the interface support the users conception of the task domain? 2) Can the questions 
be asked concisely? Designing InfoVis systems that minimize semantic distances hence requires a thorough understanding of the problem domain and tasks users want to perform.

\subsection{Model Construction and Manipulation}

The cognitive perspective that using an InfoVis system is the active construction and manipulation of a model has implications on what kind of role the user should play in this process. Traditionally it is often the case that users are given a "whole package deal": a system with predetermined representations and interaction techniques. It may be intended that in this way, users can be free from any of the design and implementation issues and focus on the tasks at hand. While it is true that user motivation and active participation are implicated in using these systems, users are cast primarily as passive interpreters and manipulators of what the system presents. In other words, the visualization model is constructed by the designers not the users, and the users have only limited freedom in transforming this model in ways predefined by the designers.

Such an approach places little burden of design and implementation on the users, but may have negative consequences. Kobsa's study for example found that Spotfire users tended to use the default scatter-plot visualization even when an alternative representation might be better [25]. While it can be argued that more training will teach users to choose the appropriate visual encodings, once users get more proficient, they may feel restricted by the system as it does not allow them to freely construct a model that does not necessarily replicate past experience. We believe this is a manifestation of a deeper question often overlooked in current InfoVis research: in model-based reasoning where an external visual model is constructed and manipulated, what roles should the designers and the users play, and what should be expected from the users?

At one end of the spectrum, there are designers who create InfoVis tools for personal use. The designers can flexibly modify and reconstruct the persistent external visualization models depending on the tasks and needs. This approach is unrealistic for average users due to the non-trivial time and effort required to possess a variety of skills such as visual design and programming. A more realistic approach, as evident in the traditional way, is to (roughly) assign the task of construction to designers and the task of manipulation to users. Users in this case do not have to be experts in visual design or programming.

While it is less controversial that the burden of programming should be eliminated whenever possible, it is not convincing that users should not worry about being able to mentally simulate alternative representations, to distinguish good visualization from the bad and to look, search and act at the right place at the right time. Using visualization to think is a cognitive skill that needs to be acquired. While training users to be familiar with predefined monolithic representations may appear sufficient for domain-specific systems, close collaboration between designers and users is too costly and time-consuming, this approach hence cannot be replicated effectively. A general-purpose approach is necessary for wider adoption of InfoVis in exploratory data analysis and sensemaking; presumably this requires higher mental simulation and visual thinking skills from the users.

Some existing work attempted to address this problem in one way or another, although sometimes implicitly. Tableau [2] builds on top of an algebraic formalism [55] and provides building blocks for different kinds of visualization in a departure from the mainstream monolithic approach. Users dynamically create visualization by dragging and dropping table columns. The Snap-together system [40, 41] explores users' ability to construct coordinated visualizations. Each of these systems afford and constrain different ways of model construction and manipulation, hence require different skills and abilities from the users. What are the advantages and disadvantages of giving users both greater control and responsibility of model construction is an interesting area to explore. This has implications on questions such as how and what is internalized as mental models, and how the interplay between internalization and externalization takes place. We currently know little about these questions, and they are worth pursuing further.

\section{IMPLICATIONS FOR EVALUATION}

\subsection{Individual Differences in the Process of Visual Rea- soning}

Existing evaluation methods, from traditional metrics such as time and error rate to more recent ones such as quantifiable insights [39], are inherently biased towards the outcomes of using InfoVis systems. From the perspective of distributed cognition and model based reasoning, however, these metrics really reflect the properties of a cognitive system comprising both the InfoVis system and the human; sometimes a larger cognitive system including the designers may be applicable too. Since no two persons are identical, individual differences should be carefully identified and differentiated from properties of InfoVis design whenever possible.

There has been no consensus on how to characterize individual differences in visual reasoning. One approach is to abstract individual differences in conceptualized measures such as visual memory, learning style and spatial cognitive ability using some standardized tests [6]. It is not clear how these abstracted measures accurately reflect a person's ability in visual thinking, and distinctions such as verbal thinker vs. visual thinker seem crude. Each individual's visual thinking ability likely will be the result of mutual influence of innate disposition and cultural upbringing, and may vary in terms of performance depending on the features of visualization and tasks at hand.

Instead of relying on standardized tests, observing and interpreting users' visual reasoning process may be a more practical approach. Based on our theories, the process of user interaction with InfoVis directly manifests how an individual performs model-based reasoning. In particular, mental simulation and physical actions are two major indicators of one's ability of using the specific given visualizations to solve the specific problems. Our formulation of the action cycles in Section 5.2 can serve as a framework for interpreting user intents behind observed physical actions. Physical actions, however, often do not provide a complete picture of the modeling process. Expert users likely will engage in intensive mental simulation as well [57]. We will discuss methodological concerns for interpreting mental simulation in the next section.

Given that both mental acts and physical acts can be analyzed, we can not only pin down individual differences in terms of visual thinking skills, but also specify the properties of visualization design. By contrasting how different users approach the same task differently, we can analyze if the success or failure of task completion is attributable to the user or the properties of design.

\subsection{How to Capture Mental Simulation}

While it is true that mental models cannot be observed directly, it is possible to hypothesize the ongoing simulative processes based on empirical data. A common approach used in cognitive science to "directly trace" concurrent thinking is the think-aloud protocol [10]. Users are asked to verbalize their thoughts as they occur. Although there are concerns regarding the protocol's potential interference with complex cognitive processes, this technique has been widely used and is shown to be effective provided that the participants do not try to explain their reasoning, but just report what they are attending to, remembering and so on. The transcribed verbal data is then coded and interpreted. In InfoVis evaluation, verbal data is often collected during interviews as direct interrogation of users' opinions about the design or their own introspection of the analytic processes; it has been less commonly used as the basis for investigating users' cognitive processes on the fly.

In a study exploring how scientists use visualizations and how they connect mental models with external visualizations, Trafton et al. interpreted verbal data and coded the number of times the scientists reason with internal models of visualizations [57]. Some might be skeptical whether verbal data can provide a close and reliable basis for inferring mental activity, but the utterances in the protocol showed quite promising results. For example, the scientists often talk about an imaginary form of visualization (e.g. "if the arm's detached here and sort of flowing away" and "in a perfect world, in a perfect sort of spider diagram"); such utterances can be interpreted with reasonably 
high confidence as evidence for simulation on a mental representation.

Of course there can be confirmation biases in interpreting verbal data. Such biases can be overcome in a number of ways: several researchers can code the verbal data independently and resolve disagreements by discussion; additional data sources such as video-tapes can be used to confirm or reject interpretations. While think-aloud protocols are often conducted as experiments in a lab-based setting, verbal analysis can be used beyond the lab in a more authentic setting too. For example, verbal analysis of the mental modeling process is applicable when expert users engage in long-term collaborations with visualization researchers [50]. Such longitudinal studies will likely accumulate historical data that can be used for more accurate interpretation.

\section{IMPLICATIONS FOR THEORY}

\subsection{Mental Models in InfoVis}

Apart from the general notion that mental models often lack item specific information and are abstracted, we know little about the properties of mental models specific in an InfoVis context. We defined mental models in InfoVis as analogue representations. It does not automatically follow, however, that mental models in InfoVis will be isomorphic to external visualizations. Assuming so likely will commit what Scaife and Rogers call "the resemblence fallacy" [48]. Tversky argued convincingly that people's mental model of geography is not necessarily visual-spatial, resembling a map [59]. When asked about the relative location of San Diego with respect to Reno, people incorrectly responded that San Diego was west of Reno. This answer is based on a mental model that is a collage of different types of information organized hierarchically. People do not remember the locations of cities. Instead they remember the relative locations of the states and infer the locations of the cities from the state superset. At the level of states, the location information might be derived from a map and is hence visual-spatial. The relationship between states and cities instead is likely to be propositional. Since raw data and data ontologies are often expressed in words in InfoVis, people's mental models of visualizations may be also in the form of collages as we have discussed earlier. What is the nature of this collage? How will visual designs affect the properties of mental models and in turn influence how these designs are being used? Investigating these questions will help us understand better how people perceive and use visualization designs in their reasoning.

\subsection{A Taxonomy of Mental Simulation?}

We suggest that mental simulation forms the basis of model-based reasoning, and interaction in InfoVis is analogous to mental simulation. What specific simulative operations do people usually perform in their mental models? If we can specify and categorize examples of simulative transformations of InfoVis, such a taxonomy may serve as a basis for interaction design. It would be also interesting to explore whether it is necessary to implement all kinds of simulative transformations as interactive features of visualization systems. For now our guess is that moving everything to the external plane is not desirable. Visualization design needs to strike a balance between physical interaction and mental simulation.

\section{Conclusion}

In this paper, we investigate the nature of internal representations relevant for InfoVis and provide a top-down account of how various aspects of human cognition are related. Our discussions suggest preliminary answers to the questions raised in the introduction:

- What is meant by the notion of 'internal representation'? We identify mental models as internal, structural, behavioral and functional analogues of external visualization systems. Mental models preserve schematic, semantic or item level information about the data, and may be in the format of collages where different types of information are overlaid.

- Given a clearer notion of internal representation, what are the relationships between external representations (visualizations) and internal representations? The dynamics between mental models and external visualizations are highly fluid. Four kinds of relationships are proposed: internalization, processing, augmentation and creation.

- How are external visualizations, people's mental faculties and physical actions related? External visualizations are internalized as mental models. Given a problem, people can construct and simulate mental models in reasoning. In the InfoVis context, external visualizations are necessary to augment mental models so that they form a coupled system. People's physical actions can be understood to achieve internal-external coupling in terms of three purposes: external anchoring, information foraging and cognitive offloading.

In addressing these questions, we set up a framework that unites visualizations, mental models, interaction and the analytic process in a coherent account. We believe this framework can guide and inform future endeavors on the design, evaluation and understanding of InfoVis systems in visual reasoning activities.

\section{ACKNOWLEDGMENTS}

We are grateful to Nancy Nersessian for the insightful discussions and comments on earlier versions of this paper. This work is supported by the National Science Foundation under award IIS-0915788 and the VACCINE Center, a Department of Homeland Security's Center of Excellence in Command, Control and Interoperability.

\section{REFERENCES}

[1] Gapminder. http://www.gapminder.org.

[2] Tableau software. http://www.tableausoftware.com/

[3] D. H. Ballard, M. M. Hayhoe, P. K. Pook, and R. P. N. Rao. Deictic codes for the embodiment of cognition. Behavioral and Brain Sciences, 20(04):723-742, 1997.

[4] R. A. Brooks. Intelligence without reason. Artificial intelligence: critical concepts, 3, 1991.

[5] S. K. Card, J. D. Mackinlay, and B. Shneiderman. Readings in information visualization: using vision to think. pages 1-34. Morgan Kaufmann Publishers Inc, Jan. 1999.

[6] C. Conati and H. Maclaren. Exploring the role of individual differences in information visualization. In Proceedings of the working conference on Advanced visual interfaces, pages 199-206, Napoli, Italy, 2008. ACM.

[7] K. Craik. The Nature of Explanation. Cambridge University Press, Cambridge, 1943

[8] A. Dix and G. Ellis. Starting simple: adding value to static visualisation through simple interaction. In Proceedings of the working conference on Advanced Visual Interfaces, pages 124-134, 1998.

[9] T. Dwyer, B. Lee, D. Fisher, K. I. Quinn, P. Isenberg, G. Robertson, C. North, and K. Inkpen. A comparison of User-Generated and automatic graph layouts. IEEE Transactions on Visualization and Computer Graphics, 15(6):961-968, 2009.

[10] K. A. Ericsson and H. A. Simon. Protocol analysis: Verbal reports as data. MIT Press, 1993.

[11] E. S. Ferguson. Engineering and the Mind's Eye. The MIT Press, 1994.

[12] J. J. Gibson. The Ecological Approach to Visual Perception. Lawrence Erlbaum Associates Inc, US, 1986.

[13] V. Gottschling. Visual imagery, mental models, and reasoning. Advances in Psychology, 138:211-235, 2006.

[14] D. Gotz and M. X. Zhou. Characterizing users visual analytic activity for insight provenance. Information Visualization, 8(1):42-55, 2009.

[15] M. Hegarty. Diagrams in the mind and in the world: Relations between internal and external visualizations. Lecture notes in computer science, pages 1-13, 2004.

[16] E. Hutchins. Material anchors for conceptual blends. Journal of Pragmatics, 37(10):1555-1577, 2005.

[17] E. L. Hutchins, J. D. Hollan, and D. A. Norman. Direct manipulation interfaces. Human-Computer Interaction, 1(4):311-338, 1985.

[18] V. John-Steiner. Notebooks of the Mind: Explorations of Thinking. Oxford University Press, USA, revised edition, 1997.

[19] P. N. Johnson-Laird. Mental models: Towards a cognitive science of language, inference and consciousness. Harvard Univ Pr, 1983. 
[20] P. N. Johnson-Laird. Imagery, visualization, and thinking. Perception and cognition at centurys end, pages 441-467, 1998.

[21] D. E. Kieras and S. Bovair. The role of a mental model in learning to operate a device. Cognitive science, 8(3):255-273, 1984.

[22] D. Kirsh. Projection, problem space and anchoring. In Proceedings of the 31 st Annual Conference of the Cognitive Science Society, pages 2310 2315, 2009.

[23] G. Klein, J. K. Phillips, E. L. Rall, and D. A. Peluso. A data-frame theory of sensemaking. In Expertise Out of Context: Proc. 6th Intl Conf. Naturalistic Decision Making, 2006.

[24] M. Knauff and P. N. Johnson-Laird. Visual imagery can impede reasoning. Memory \& Cognition, 30(3):363-371, 2002.

[25] A. Kobsa. An empirical comparison of three commercial information visualization systems. In IEEE Symposium on Information Visualization, pages 123-130, 2001.

[26] S. M. Kosslyn, W. L. Thompson, and G. Ganis. The case for mental imagery. Oxford University Press, USA, 2006.

[27] G. Lakoff and M. Johnson. Metaphors We Live by. University of Chicago Press, 1980

[28] H. Lam. A framework of interaction costs in information visualization. IEEE transactions on visualization and computer graphics, 14(6):1149_ $1156,2008$.

[29] J. H. Larkin and H. A. Simon. Why a diagram is (sometimes) worth ten thousand words. Cognitive Science, 11(1):65-100, 1987.

[30] Z. Liu, N. J. Nersessian, and J. T. Stasko. Distributed cognition as a theoretical framework for information visualization. IEEE Transactions on Visualization and Computer Graphics, 14(6):1173-1180, Dec. 2008.

[31] Z. Liu, J. Stasko, and T. Sullivan. SellTrend: Inter-Attribute visual analysis of temporal transaction data. IEEE Transactions on Visualization and Computer Graphics, 15(6):1025-1032, 2009.

[32] R. H. Logie. Visuo-spatial working memory. Psychology Press, 1995.

[33] L. Magnani and N. J. Nersessian. Model-based reasoning: science, technology, values. Springer, 2002.

[34] J. M. Mandler. Stories, Scripts, and Scenes: Aspects of Schema Theory. Psychology Press, 1st edition, 1984.

[35] B. A. Nardi and C. L. Zarmer. Beyond models and metaphors: Visual formalisms in user interface design. Journal of Visual Languages \& Computing, 4(1):5-33, 1993.

[36] N. Nersessian. Creating scientific concepts. The MIT Press, 2008.

[37] A. Noe. Action in Perception. The MIT Press, 2005.

[38] D. A. Norman. Some observations on mental models. Mental models, pages 7-14, 1983.

[39] C. North. Visualization viewpoints: Toward measuring visualization insight. IEEE Computer Graphics and Applications, 26(3):6-9, 2006.

[40] C. North and B. Shneiderman. Snap-together visualization: a user interface for coordinating visualizations via relational schemata. In Proceedings of the working conference on Advanced visual interfaces, pages $128-135,2000$.

[41] C. North and B. Shneiderman. Snap-together visualization: can users construct and operate coordinated visualizations? International Journal of Human Computer Studies, 53(5):715-740, 2000.

[42] S. J. Payne. Users mental models: The very ideas. HCI Models, theories and frameworks: Toward a multidisciplinary science, pages 135156, 2003.

[43] W. A. Pike, J. Stasko, R. Chang, and T. A. O'Connell. The science of interaction. Information Visualization, 8(4):263-274, 2009.

[44] P. Pirolli and S. Card. The sensemaking process and leverage points for analyst technology as identified through cognitive task analysis. In Proceedings of International Conference on Intelligence Analysis, pages 2-4, 2005.

[45] H. Purchase, E. Hoggan, and C. Görg. How important is the "Mental map"? an empirical investigation of a dynamic graph layout algorithm. In Graph Drawing, pages 184-195, 2006.

[46] Z. W. Pylyshyn. What the mind's eye tells the mind's brain: A critique of mental imagery. Psychological bulletin, 80(1):1-24, 1973.

[47] W. B. Rouse and N. M. Morris. On looking into the black box: Prospects and limits in the search for mental models. Psychological bulletin, 100(3):349-363, 1986

[48] M. Scaife and Y. Rogers. External cognition : how do graphical representations work? Int. J. Human-Computer Studies, 45(2):185-213, 1996.

[49] B. Shneiderman. The eyes have it: A task by data type taxonomy of information visualizations. In Proc. IEEE Visual Languages '96, pages 336-343, 1996.
[50] B. Shneiderman and C. Plaisant. Strategies for evaluating information visualization tools: Multi-dimensional in-depth long-term case studies. In BELIV : BEyond time and errors: novel evaLuation methods for Information Visualization, Venice, Italy, 2006.

[51] B. Shore. Culture in Mind: Cognition, Culture, and the Problem of Meaning. Oxford University Press US, 1996.

[52] R. Spence. Information Visualization: Design for Interaction. Prentice Hall, 2nd edition, 2007.

[53] D. Sperber. Anthropology and psychology: Towards an epidemiology of representations. Man, 20(1):73-89, 1985.

[54] M. Spivey. The Continuity of Mind. Oxford University Press, USA, 2008.

[55] C. R. Stolte. Query, analysis, and visualization of multidimensional databases. PhD thesis, Stanford University, 2003.

[56] J. J. Thomas and K. A. Cook. Illuminating the path: The research and development agenda for visual analytics. IEEE Computer Society, 2005.

[57] J. G. Trafton, S. B. Trickett, and F. E. Mintz. Connecting internal and external representations: Spatial transformations of scientific visualizations. Foundations of Science, 10(1):89-106, 2005.

[58] E. R. Tufte. Visual Explanations: Images and Quantities, Evidence and Narrative. Graphics Press, 4th printing with revisions edition, Feb. 1997.

[59] B. Tversky. Cognitive maps, cognitive collages, and spatial mental models. Spatial Information Theory - A Theoretical Basis for GIS: Proc. European Conference COSIT, pages 14-24, 1993.

[60] L. Tweedie. Characterizing interactive externalizations. In Proceedings of the SIGCHI conference on Human factors in computing systems, pages 375-382, 1997.

[61] F. van Ham and B. Rogowitz. Perceptual organization in user-generated graph layouts. IEEE Transactions on Visualization and Computer Graphics, 14(6):1333-1339, 2008.

[62] L. S. Vygotsky. Thought and Language. The MIT Press, 1986.

[63] T. D. Wang, C. Plaisant, B. Shneiderman, N. Spring, D. Roseman, G. Marchand, V. Mukherjee, and M. Smith. Temporal summaries: Supporting temporal categorical searching, aggregation and comparison. IEEE Transactions on Visualization and Computer Graphics, 15(6):1049-1056, 2009.

[64] J. V. Wertsch. Vygotsky and the Social Formation of Mind. Harvard University Press, 1988.

[65] L. Wilkinson. The grammar of graphics. Springer Verlag, 2005.

[66] J. S. Yi, Y. ah Kang, J. T. Stasko, and J. A. Jacko. Toward a deeper understanding of the role of interaction in information visualization. IEEE Transactions on Visualization and Computer Graphics, 13(6):1224 1231, 2007.

[67] C. Ziemkiewicz and R. Kosara. The shaping of information by visual metaphors. IEEE Transactions on Visualization and Computer Graphics, 14(6), Dec. 2008. 